\title{
Development and identification of three functional markers associated with starch content in lotus (Nelumbo nucifera)
}

Teng Cheng ${ }^{1}$, Xingwen Zheng ${ }^{1}$, Keqiang Xie ${ }^{2}$, Jiangdong Liu ${ }^{3}$, Xingfei Zheng ${ }^{1}$, Surong Jin $^{4}$, Ying Diao ${ }^{1,5}$, Zhongli $\mathrm{Hu}^{1^{*}}$ \& Jianxiong Wang ${ }^{1 *}$

It have been significantly demonstrated that Hexokinase (HXK), Granule-bound starch synthase (GBSS) and ADP-glucose pyrophosphorylase (AGPase) are three critical enzymes in the starch biosynthetic pathway and are related to starch (amylose, amylopectin and total starch) content in lotus. It is important to develop functional markers in marker-assisted selection of lotus breeding. So far there have been few reports about lotus functional markers. In this study, based on insertion-deletions (INDELs) and single-nucleotide polymorphisms (SNPs), we developed three functional markers, FMHXK-E1, FMGBSS-18 and FMAGPL-I1. FMHXK-E1 was developed based on polymorphisms of two haplotypes of NnHXK. 26 lotus cultivars that the 320-bp fragment presented in $\mathrm{NnHXK}$ had a lower content of amylose and a higher content of amylopectin. FMGBSS-18 was developed based on polymorphisms of two haplotypes of NnGBSS. The group containing 32 lotus cultivars with the 210-bp fragment had less amylose content and more amylopectin content. FMAGPL-I1 was developed based on polymorphisms of two haplotypes of NnAGPL (ADP-glucose pyrophosphorylase large subunit gene). The group containing 40 lotus cultivars with the 362 -bp fragment had less amylopectin, total starch content and more amylose content. According to the study, FMHXK-E1, FMGBSS-18 and FMAGPL-I1 are closely related to lotus starch content. It could be provided research basis for molecular assisted selection of lotus starch content improve breeding efficiency.

Lotus (Nelumbo nucifera Gaertn), a perennial aquatic herb, is one of the oldest dicotyledonous plants ${ }^{1}$, which originated and has been widely grown in southern China for thousands of years ${ }^{2}$. Nelumbo Adans, a surviving living fossil that has experienced the Quaternary glacial period, has an evolutionary history of almost 135 million years. In addition to the evolution values, lotus is also a kind of essential traditional Chinese medicine and food and its rhizome has been widely consumed for over 7000 years in Asia ${ }^{3}$. Starch content is one of the main factors affecting the lotus root processing and cooking quality. Starch can be divided into amylose and amylopectin in plant ${ }^{4}$. Amylose is a 200 glucose groups polysaccharide linear molecule with glucose residues linked together by $\alpha-(1,4)$ glycosidic bonds. Amylopectin is a 300 to 400 glucose groups chain molecule linked together by $\alpha-(1,4)$ glycosidic bonds and $\alpha-(1,6)$ glycosidic bonds ${ }^{5,6}$.

Identifying genes and molecular markers associated with trait variation is obligatory for comprehending molecular breeding and crop improvement ${ }^{7}$. Up to now it is possible to develop markers from genes that have a putative function which is referred to as 'functional markers' $(\mathrm{FMs})^{8}$. FMs that developed from gene polymorphisms affect phenotypic trait variation ${ }^{8,9}$. Therefore, it is necessary to understand the function of genes in the development of functional markers. In recent years, the new type of molecular marker (FM), based on the insertion/ deletion (INDEL) and the single-nucleotide polymorphism (SNP), has been successfully developed and play a broader role in plant molecular marker-assisted breeding ${ }^{10-12}$. For example, the functional marker of GBSS

\footnotetext{
${ }^{1}$ State Key Laboratory of Hybrid Rice, Hubei Lotus Engineering Center, College of Life Sciences, Wuhan University, Wuhan, 430072, P. R. China. ${ }^{2}$ Guangchang Bailian Institute of Jiangxi Province, Guangchang, 344900, P. R. China. ${ }^{3}$ College of Life Sciences, Wuhan University, Wuhan, 430072, P. R. China. ${ }^{4}$ School of Chemistry, Chemical Engineering and Life Science, Wuhan University of Technology, Wuhan, 430070, P. R. China. ${ }^{5}$ College of Forestry and Life Sciences, Chongqing University of Arts and Sciences, Yongchuan, 402160, China. *email: huzhongli@whu.edu.cn; 214065995@qq.com
} 


\begin{tabular}{|c|c|c|c|c|c|}
\hline $\begin{array}{l}\text { Locus (accession Number } \\
\text { or reference) }\end{array}$ & Encoded protein & $\begin{array}{l}\text { Primer } \\
\text { name }\end{array}$ & $\begin{array}{l}\text { Primer sequence }\left(5^{\prime}-3^{\prime}\right) \\
\text { or reference }\end{array}$ & $\begin{array}{l}\text { PCR product } \\
\text { size (bp) }\end{array}$ & $\begin{array}{l}\text { Annealing } \\
\text { temperature }\left({ }^{\circ} \mathrm{C}\right)\end{array}$ \\
\hline \multirow{2}{*}{ The first exon of $H X K$} & \multirow{2}{*}{ Hexokinase } & \multirow{2}{*}{ HXK-E1 } & f-tct aaa tcc caa tcc gtc c & \multirow{2}{*}{$308+320$} & \multirow{2}{*}{51} \\
\hline & & & r-gca cga act ctt ggc aat c & & \\
\hline \multirow{2}{*}{ The eighth intron of GBSS } & \multirow{2}{*}{$\begin{array}{l}\text { Granule-bound starch } \\
\text { synthase }\end{array}$} & \multirow{2}{*}{ GBSS-I8 } & f-ggc att act ggt att gtg a & \multirow{2}{*}{$210+220$} & \multirow{2}{*}{53} \\
\hline & & & r-gct tcc ttt aga aga ggc t & & \\
\hline \multirow{3}{*}{ The first intron of $A G P L$} & \multirow{3}{*}{$\begin{array}{l}\text { ADP-glucose phosphorylase } \\
\text { large subunit }\end{array}$} & \multirow{3}{*}{ AGPL-I1 } & f-tgg att ctt gtt gtg cga c & \multirow{3}{*}{$0+362$} & \multirow{3}{*}{57} \\
\hline & & & r1-tgg aaa gaa tag cct ggg & & \\
\hline & & & r2-tgg aaa gaa tag cct gtg & & \\
\hline
\end{tabular}

Table 1. Locus and Primer information.

has been used to select wheat materials, which is linked with flour quality ${ }^{13}$. Although traditional SSR and ISSR analysis of genetic diversity have been used in lotus cultivars ${ }^{14-16}$, functional markers associated with starch content have not been developed and applied in lotus cultivars. So, it is a critical step towards selecting suitable lotus cultivars to develop functional markers on the identification of amylose, amylopectin and total starch content.

Starch is only produced through biosynthetic pathway, which involves lots of conservative function enzymes, such as hexokinase (HXK), granule-bound starch synthase (GBSS), ADP-glucose phosphorylase (AGP), soluble starch synthases (SSS), starch branching enzymes (SBE), starch debranching enzymes (DBE) and so on ${ }^{4,17}$. Numerous studies showed that granule-bound starch synthase was encoded by the GBSS gene, which catalyzes amylose synthesis ${ }^{18-20}$. AGP is a rate-limiting enzyme which catalyzes ATP and Glc-1-P to pyrophosphate and ADP-glucose (ADPG). ADPG acts as the substrate for the synthesis of amylose and amylopectin under the action of others starch synthases ${ }^{21-23}$. HXK could provide a carbon stream for plant starch synthesis which catalyzes fructose to Glc-1- $\mathrm{P}^{6}$. Identifying genes that control starch content could contribute to explore molecular markers about starch content.

In this study, 10 genes, hexokinase gene $(H X K)$, granule-bound starch synthase gene (GBSS), ADP-glucose phosphorylase gene $(A G P)$, soluble starch synthases gene $(S S S)$, starch branching enzymes gene (SBE), sucrose synthase gene ( $S U S Y)$, fructokinase gene (FRK), UDP glucose pyrophosphrylase gene (UGP), isoamylase gene $(I S A)$ and pullulanase gene (PUL), were employed to develop functional markers. Our group have resequenced to $>13 \times$ raw data of 69 lotus accessions (accession no. SRP095218). On the basis of these genomic data and the starch biosynthetic pathway, the SNP and Indel were identified in lotus. We validated the Indel and SNP in 46 different cultivars and further analysis revealed that three enzymes, HXK, GBSS and AGPL, significantly associated with starch content are encoded by Indel or SNP of genes. Finally, we respectively developed three functional markers, FMHXK-E1, FMGBSS-I8 and FMAGPL-I1, from HXK, GBSS and AGPL.

\section{Results}

Development and identification of FMHXK-E1. The $320 \mathrm{bp}$ and $308 \mathrm{bp}$ fragment sequences of $\mathrm{NnHXK}$ were detected by primer HXK-1E (Table 1). An inserted/deleted fragment of 12-bp was found in the exon of NnHXK by blasting result of PCR product sequencing (Fig. 1). Based on the results of PCR detection, a pair of alleles with 308-bp and 320-bp fragment were detected (Supplementary File A). In order to investigate the effect of the 12-bp Indel on starch content, 320-bp fragment differences in 46 lotus accessions were analyzed. It was presented by Excel analysis that the amylose content is lower and the amylopectin content is higher of 26 lotus cultivars with the 320-bp fragment of $N n H X K$, and the amylose content is higher and the amylopectin content is lower of the another 20 lotus cultivars without the 320-bp fragment of NnHXK. The percentage of amylose in dry matter (5.23\%) and the percentage in total starch $(22.09 \%)$ are both significantly higher than the percentage of amylopectin in dry matter (3.13\%) and the percentage in total starch (11.53\%). Correlation analysis showed that the significance differences of amylose and amylopectin content in total starch with diversity bands of marker reached a high level. In FMHXK-E1, with diversity bands of marker, correlation analysis showed significant differences in amylose and amylopectin content in total starch that $\mathrm{P}$ value reached a high level at 0.010 and $0.008(\mathrm{P} \leqq 0.01)$ respectively. $\mathrm{P}$ value reached a very level at $0.007(\mathrm{P} \leqq 0.01)$ of amylose content in dry matter (Table 2). The functional marker was developed and named FMHXK-E1 according to the gene HXK and primer.

Development and identification of FMGBSS-18. The 210-bp and 220-bp fragment sequences of $N n G B S S$ were detected by primer GBSS-8I (Table 1). An inserted/deleted fragment of 10-bp was found in the intron of NnGBSS by blasting result of PCR product sequencing (Fig. 2). A pair of alleles with 210-bp and 220-bp fragment were detected according to the results of PCR detection (Supplementary File B). In order to explore the influence of the 10-bp Indel on starch content, 210-bp fragment differences in 46 lotus cultivars were analyzed. According to the result of t-test analysis, it was showed that 32 lotus cultivars with the $210 \mathrm{bp}$ fragment of $N n G B S S$ had lower amylose content and higher amylopectin content and another 14 lotus cultivars missing the 210-bp fragment of NnGBSS contained more amylose and less amylopectin. The amylose percentage in dry matter $(5.26 \%)$ and in total starch $(23.56 \%)$ of 14 lotus cultivars missing the 210 -bp fragment are both higher than the amylopectin percentage in dry matter $(3.51 \%)$ and in total starch (13.09\%) of 32 lotus cultivars with the $210 \mathrm{bp}$ fragment. Apparently, there was a significant correlation between the PCR bands diversity and starch (amylose and amylopectin) content. In FMGBSS II-1, with diversity bands of marker, correlation analysis showed significant differences in amylose and amylopectin content in total starch that $\mathrm{P}$ value at 0.040 and $0.022(\mathrm{P} \leqq 0.05)$ respectively. The $\mathrm{P}$ value at $0.026(\mathrm{P} \leqq 0.05)$ of amylose content in dry matter (Table 3$)$. The functional marker was developed and named FMGBSS-I8 according to the gene GBSS and primer. 


\section{A 12-bp InDel of $\mathrm{NnHXK}$}

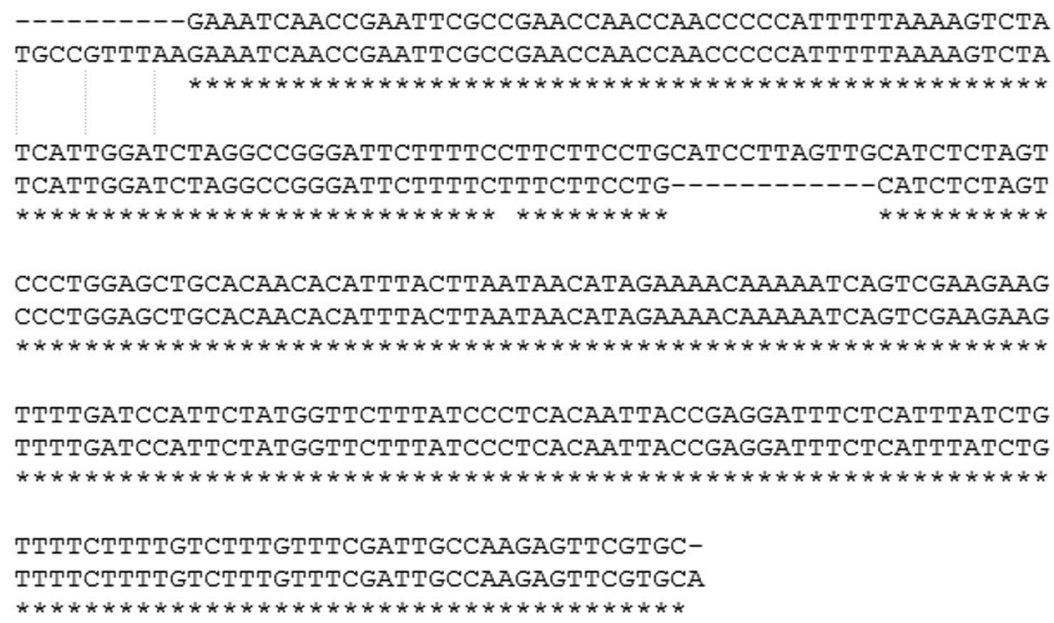

Figure 1. Indel of $N n H X K$. The 12-bp InDel region in first exon of $N n H X K$ is indicated in the white background.

A 10-bp Indel of NnGBSS

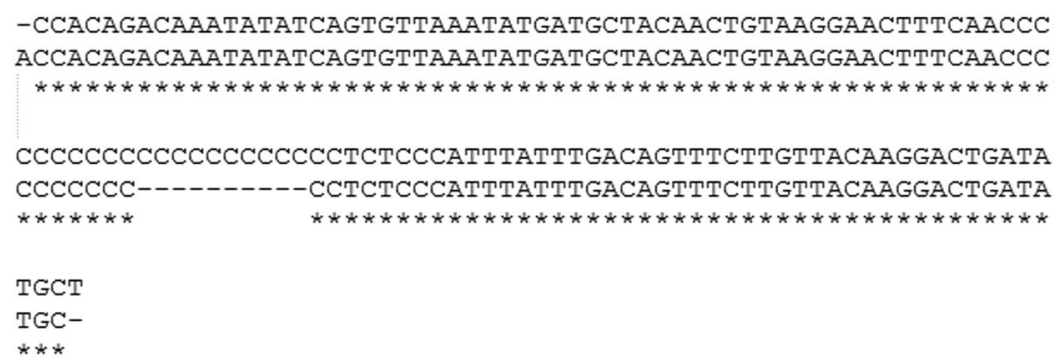

Figure 2. Indel of NnGBSS. There is a 10-bp InDel in the eighth intron of NnGBSS II-1. The 10-bp InDel region in the eighth intro of NnGBSS II-1 is indicated in white background.

\begin{tabular}{|l|l|l|l|l|l|l|}
\hline No. & item & $\begin{array}{l}\text { PCR products without } \\
\mathbf{3 2 0} \text { bp fragment }\end{array}$ & $\begin{array}{l}\text { PCR products with } \\
\mathbf{3 2 0} \text { bp fragment }\end{array}$ & t value & p value & $\begin{array}{l}\text { significance } \\
\text { level }\end{array}$ \\
\hline 1 & $\begin{array}{l}\text { the percentage of amylose in } \\
\text { dry matter, \% }\end{array}$ & 5.23 & 3.13 & 2.83 & 0.007 & $* *$ \\
\hline 2 & $\begin{array}{l}\text { the percentage of amylopectin } \\
\text { in total starch, } \%\end{array}$ & 77.89 & 88.08 & -2.67 & 0.010 & $* *$ \\
\hline 3 & $\begin{array}{l}\text { the percentage of amylose in } \\
\text { total starch, \% }\end{array}$ & 22.09 & 11.53 & 2.76 & 0.008 & $* *$ \\
\hline 4 & $\begin{array}{l}\text { the percentage of amylopectin } \\
\text { in dry matter, } \%\end{array}$ & 23.94 & 30.70 & -1.59 & 0.119 & NS \\
\hline 5 & $\begin{array}{l}\text { the percentage of total starch } \\
\text { in dry matter, } \%\end{array}$ & 29.18 & 33.83 & -1.16 & 0.254 & NS \\
\hline
\end{tabular}

Table 2. The results of t-test FMHXK-E1. **Indicated that the difference reached a very significant level,

*Indicated that the difference reached a significant level, "NS" indicated that the difference was not significant.

Development and identification of FMAGPL-I1. A SNP of C/A sequences of NnAGPL was found in the first intron of NnAGPL by primer AGPLI1 (Fig. 3) (Table 1). According to the principle of ARMS (The amplification refractory mutation system $)^{24-27}$, AGPLI1 had the same forward primer, and the second bases of $3^{\prime}$-reverse primers were complementary each other. Through forward primer-f and reverse primer-r 1 , PCR amplification yielded a 362-bp fragment in 40 lotus cultivars. The other 6 lotus cultivars lacked the 362-bp fragment, because the mutant carried a point mutation in the intron of NnAGPL, where base corresponded to the second base of $3^{\prime}$-reverse primer (Supplementary File C). In order to study the effect of the mutant on starch content, 210-bp fragment differences in 46 lotus cultivars were analyzed. The total starch content in the lotus with SNP site at C base was lower (total starch accounted for $30.2 \%$ in dry matter), while the total starch content in the lotus with SNP site at A base was higher (total starch accounted for $42.23 \%$ in dry matter) According to the analysis of 


\begin{tabular}{|l|l|l|l|l|l|l|}
\hline No. & item & $\begin{array}{l}\text { PCR products without } \\
\text { 210 bp fragment }\end{array}$ & $\begin{array}{l}\text { PCR products with } \\
\text { 210 bp fragment }\end{array}$ & t value & p value & $\begin{array}{l}\text { significance } \\
\text { level }\end{array}$ \\
\hline 1 & $\begin{array}{l}\text { the percentage of amylose in dry } \\
\text { matter, \% }\end{array}$ & 5.26 & 3.51 & -2.11 & 0.040 & $*$ \\
\hline 2 & $\begin{array}{l}\text { the percentage of amylopectin in } \\
\text { total starch, \% }\end{array}$ & 76.94 & 86.59 & 2.31 & 0.026 & $*$ \\
\hline 3 & $\begin{array}{l}\text { the percentage of amylose in } \\
\text { total starch, \% }\end{array}$ & 23.56 & 13.09 & -2.37 & 0.022 & $*$ \\
\hline 4 & $\begin{array}{l}\text { the percentage of amylopectin in } \\
\text { dry matter, \% }\end{array}$ & 22.26 & 30.17 & 1.74 & 0.089 & NS \\
\hline 5 & $\begin{array}{l}\text { the percentage of total starch in } \\
\text { dry matter, \% }\end{array}$ & 27.41 & 33.54 & 1.43 & 0.171 & NS \\
\hline
\end{tabular}

Table 3. The results of t-test FMGBSS-I8. *Indicated that the difference reached a significant level, NS indicated that the difference was not significant.

\begin{tabular}{|l|l|l|l|l|l|l|}
\hline No. & item & $\begin{array}{l}\text { PCR products without } \\
\mathbf{3 6 2} \mathbf{~ b} \text { fragment }\end{array}$ & $\begin{array}{l}\text { PCR products with } \\
\mathbf{3 6 2} \mathbf{b} \text { f fragment }\end{array}$ & t value & p value & $\begin{array}{l}\text { significance } \\
\text { level }\end{array}$ \\
\hline 1 & $\begin{array}{l}\text { the percentage of amylose in dry } \\
\text { matter, } \%\end{array}$ & 25.90 & 40.20 & 2.36 & 0.023 & $*$ \\
\hline 2 & $\begin{array}{l}\text { the percentage of amylopectin in } \\
\text { total starch, } \%\end{array}$ & 4.35 & 2.04 & -2.03 & 0.048 & $*$ \\
\hline 3 & $\begin{array}{l}\text { the percentage of amylose in } \\
\text { total starch, \% }\end{array}$ & 30.24 & 42.23 & 2.09 & 0.042 & $*$ \\
\hline 4 & $\begin{array}{l}\text { the percentage of amylopectin in } \\
\text { dry matter, \% }\end{array}$ & 82.05 & 94.30 & 2.12 & 0.039 & $*$ \\
\hline 5 & $\begin{array}{l}\text { the percentage of total starch in } \\
\text { dry matter, \% }\end{array}$ & 17.68 & 5.71 & -2.06 & 0.045 & $*$ \\
\hline
\end{tabular}

Table 4. The results of t-test FMAGPL-I1. *Indicated that the difference reached a significant level.

A SNP C/A of NnAGPL

ATTTACACAGGCTATTCTTTCCATTTCATTTTCCAATTTGAAAGATGAACTGGTTCGGTA -----CACAGGCTATTCTTTCCA----------------------------------ATTTACCCAGGCTATTCTTTCCATTTCATTTTCCAATTTGAAAGATGAACTGGTTCGGTA

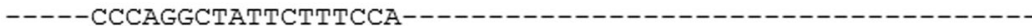
$* * * * * * * * * * * * * * * * *$

Figure 3. SNP of NnAGPL. A SNP C/A in the first intron of NnAGPL. The second and fourth lines are complementary sequences of $\mathrm{r}-2$ and $\mathrm{r}-1$, respectively.

t-test, the results showed that the polymorphism of NnAGPL was significantly correlated with the starch content (Table 4). Association analysis showed that total starch had a significant difference $(\mathrm{P}=0.042)$ with fragment polymorphism $(\mathrm{P} \leq 0.05)$. FMAGPL-I1 could directly screen out lotus varieties with high or low total starch content. The functional marker was developed and named FMAGPL-I1 according to the gene AGPL and primer name.

\section{Discussion}

Functional markers is a novel DNA molecular markers, which were developed from polymorphic motifs of functional genes causing differences in phenotypic traits. The association analysis of plant population phenotypic characteristics and functional genes of phenotypic correlation is a method to develop indirect functional markers. With the separation and annotation of functional genes, functional markers are gradually becoming a new type of DNA molecular markers since random DNA molecular markers, which can greatly improve the efficiency and accuracy of labeling. Compared with the other DNA molecular markers, functional markers have a broad application prospect in assisted genetic breeding and variety identification, because they are completely related to polymorphism sequences of functional genes. Iyer-pascuzzi and McCouch developed functional markers from functional single nucleotide polymorphisms of $x a-5$, the gene of rice blast disease resistant, which can quickly and accurately select the rice varieties with resistance to rice blast disease and greatly improve the breeding process ${ }^{28}$.

There was the explanation allelic variation at some loci might indeed be causal for the trait variation for this finding. And INDELs or SNPs polymorphisms were performed to significantly affect characters of agronomic ${ }^{29}$. The possible molecular mechanism was that amino acid changes had contributed to the variation of enzyme activity, stability or post-translational modifications, which altered protein conformation or modification sites, or the variation in expression level due to DNA polymorphisms in cis-regulatory sequences. In previous studies on wheat phytoene synthase 1 gene (Psy1), a 37-bp insertion in the second intron exhibited significant association with wheat Psy1 (phytoene synthase 1) activity ${ }^{30}$. Similar study had certificated SNP A/G polymorphisms significant associated with Dhn1 and Rsp41 activity for the drought resistance ${ }^{10}$. 


\begin{tabular}{|c|c|c|c|c|c|c|c|}
\hline No. & Name & Sources & $\begin{array}{l}\text { the percentage of } \\
\text { amylopectin in } \\
\text { dry matter, \% }\end{array}$ & $\begin{array}{l}\text { the percentage } \\
\text { of amylose in } \\
\text { dry matter, \% }\end{array}$ & $\begin{array}{l}\text { the percentage } \\
\text { of total starch in } \\
\text { dry matter, \% }\end{array}$ & $\begin{array}{l}\text { the percentage of } \\
\text { amylopectin in } \\
\text { total starch, } \%\end{array}$ & $\begin{array}{l}\text { the percentage } \\
\text { of amylose in } \\
\text { total starch, \% }\end{array}$ \\
\hline 1 & Thailand lotus-I & Thailand & 20.4 & 2.9 & 23.3 & 87.55 & 12.45 \\
\hline 2 & Cunsan lotus & Xiangtan, Hunan & 14.63 & 4.48 & 19.11 & 76.54 & 23.45 \\
\hline 3 & Baixiang lotus & Xiangtan, Hunan & 16.71 & 3.78 & 20.49 & 81.55 & 18.46 \\
\hline 4 & Hongxiang lotus & Xiangtan, Hunan & 5.38 & 2.63 & 8.01 & 67.18 & 32.82 \\
\hline 5 & Dongtinghu wild lotus & Dongtinghu, Hunan & 51.01 & 6.02 & 57.03 & 89.43 & 10.57 \\
\hline 6 & Nangeng wild lotus & Ma’anshan, Anhui & 18.08 & 10.95 & 29.03 & 62.27 & 37.73 \\
\hline 7 & Tuxuan lotus & Jinhua, Zhejiang & 28.43 & 5.26 & 33.69 & 84.38 & 15.32 \\
\hline 8 & Chuzhoubai lotus & Jinhua, Zhejiang & 13.12 & 9.09 & 22.21 & 59.08 & 40.92 \\
\hline 9 & Baihuajian lotus & Jinhua, Zhejiang & 34.39 & 3.53 & 37.92 & 90.69 & 9.31 \\
\hline 10 & Honghuajian lotus & Jinhua, Zhejiang & 22.54 & 6.13 & 28.67 & 78.63 & 21.37 \\
\hline 11 & Baiye lotus & Guangchang, Jiangxi & 30.87 & 4.56 & 35.43 & 87.13 & 12.87 \\
\hline 12 & Jingguang-2 hao & Guangchang, Jiangxi & 9.09 & 3.89 & 12.98 & 70.01 & 29.91 \\
\hline 13 & Xingkongmudan & Guangchang, Jiangxi & 14.71 & 2.86 & 17.57 & 83.71 & 16.29 \\
\hline 14 & Gudai lotus & Wuhan, Hubei & 45.41 & 2.29 & 47.7 & 95.19 & 4.81 \\
\hline 15 & Zhongnanhaigu lotus & Wuhan, Hubei & 60.86 & 3.53 & 64.39 & 94.51 & 5.49 \\
\hline 16 & Thailand lotus-VI & Thailand & 25.99 & 2.21 & 28.2 & 92.15 & 7.85 \\
\hline 17 & Puzheheibai lotus & Wuhan, Hubei & 29.29 & 3 & 32.29 & 90.70 & 9.3 \\
\hline 18 & Zhanhongtu & Beijing & 27.13 & 8.16 & 35.29 & 76.87 & 23.13 \\
\hline 19 & Baiyangdianhong lotus & Nanjing, Jiangsu & 36.74 & 1.02 & 37.76 & 97.31 & 2.69 \\
\hline 20 & Zhuanshang lotus & Beijing & 34.9 & 8.43 & 43.33 & 80.55 & 19.45 \\
\hline 21 & Jiandehonghua lotus & Jiande, Zhejiang & 12.94 & 9.67 & 22.61 & 57.24 & 42.76 \\
\hline 22 & Sumudan & Guangchang, Jiangxi & 34.23 & 1.01 & 35.24 & 97.14 & 2.86 \\
\hline 23 & Taikong 36 hao & Guangchang, Jiangxi & 33 & 1.9 & 34.9 & 94.54 & 5.46 \\
\hline 24 & Jingguang-2 hao & Guangchang, Jiangxi & 25.47 & 2.88 & 28.35 & 89.82 & 10.18 \\
\hline 25 & Jianxuan-17 hao & Jianning, Fujian & 44.75 & 1.52 & 46.27 & 96.70 & 3.3 \\
\hline 26 & Thailand lotus-III & Thailand & 14.84 & 2.93 & 17.77 & 83.52 & 16.48 \\
\hline 27 & Thailand lotus-IV & Thailand & 8.81 & 8.74 & 17.55 & 50.19 & 49.81 \\
\hline 28 & Thailand lotus- $\mathrm{V}$ & Thailand & 46.37 & 1.79 & 48.16 & 96.29 & 3.71 \\
\hline 29 & Jianxuan-35 hao & Jianning, Fujian & 6.87 & 10.53 & 17.4 & 39.48 & 60.52 \\
\hline 30 & Jinfurong-2 hao & Jinhua, Zhejiang & 15.99 & 6.35 & 22.34 & 71.56 & 28.44 \\
\hline 31 & Jianxuan-30 hao & Jianning, Fujian & 7.6 & 3.58 & 11.18 & 67.98 & 32.02 \\
\hline 32 & Changezuiwu & Guangchang, Jiangxi & 33.39 & 1.8 & 35.19 & 94.88 & 5.12 \\
\hline 33 & Taikongjiaorong & Guangchang, Jiangxi & 46.01 & 3.7 & 49.71 & 92.56 & 7.44 \\
\hline 34 & Fengjuanhongqi & Guangchang, Jiangxi & 24.32 & 3.25 & 27.57 & 88.19 & 11.81 \\
\hline 35 & Cuihehongyan & Guangchang, Jiangxi & 16.78 & 3.08 & 19.86 & 84.47 & 5.53 \\
\hline 36 & Lvguozijuan & Guangchang, Jiangxi & 23.97 & 4.2 & 28.17 & 85.09 & 14.91 \\
\hline 37 & Baihu wild lotus & Anhui & 22.37 & 5.79 & 28.16 & 79.45 & 20.55 \\
\hline 38 & Shanmiao wild lotus & Anhui & 15.55 & 2 & 17.55 & 88.62 & 11.39 \\
\hline 39 & Gan-62 hao & Guangchang, Jiangxi & 31.04 & 3.58 & 34.62 & 89.65 & 10.35 \\
\hline 40 & Donggua lotus & Changsha, Hunan & 36.41 & 1.14 & 37.55 & 96.95 & 3.05 \\
\hline 41 & Qingtang wild lotus & Anhui & 53.08 & 1.84 & 54.92 & 96.64 & 3.36 \\
\hline 42 & Diaochahu lotus & Hanchuan, Hubei & 56.01 & 1.54 & 57.55 & 97.32 & 2.68 \\
\hline 43 & Yixian lotus & Nanjing, Jiangsu & 16.79 & 3.99 & 20.78 & 80.79 & 19.21 \\
\hline 44 & Wuxi lotus & Wuxi, Jiangsu & 48.42 & 1.29 & 49.71 & 97.40 & 2.6 \\
\hline 45 & Furong lotus & Xiangtan, Hunan & 16.71 & 2.2 & 18.91 & 88.36 & 11.64 \\
\hline 46 & Lianhu wild lotus & Anhui & 45.68 & 1.08 & 45.76 & 97.69 & 2.31 \\
\hline
\end{tabular}

Table 5. The information of lotus samples and starch content used in this study.

At present, conventional breeding methods are inefficient in improving the starch traits of lotus roots. For this case, functional markers developed from genes that control enzymes involved in starch, is more wisely to select plants in the seedling stage. It can accelerate genetic breeding process by improving the lotus root quality and traits. The experiment of this study is an association tests for starch content traits in advanced cultivars and breeding materials of lotus root. Three functional markers, FMHXK-E1, FMGBSS-I8 and FMAGPL-I1, were developed based on sequence polymorphism among genotypes. In FMHXK-E1 and FMGBSS-I8 markers, amylose and the amylopectin content in the dry matter and total starch have significant differences with fragment polymorphism, but they are different in degree. The difference between FMHXK-E1 and starch content is extremely significant $(\mathrm{P} \leq 0.01)$, 
FMGBSS-I8 and starch content are significantly different $(\mathrm{P} \leq 0.05)$. In FMAGPL, total starch in dry matter had a significant difference $(\mathrm{P}=0.042)$ with fragment polymorphism. All of the loci were selected based on co-localization of functional candidate genes for starch content ${ }^{31-33}$. Target lotus root with suitable starch content could be selected from different lotus root cultivars and our results illustrated these functional markers were credible as reliable markers for molecular assisted selection of lotus starch content. The markers are closely linked to the target genes. Compared to the traditional way that measuring the starch content of rhizomes in the later stage of lotus growth, it provides the possibility for selection in the early stage and single plant can be selected as the test object. It can greatly reduce the blindness of the breeding process, shorten the breeding life and improve breeding efficiency.

\section{Materials and Methods}

Test materials and genomic DNA extration. 46 different cultivars of lotus roots were provided by the Guangchang Bailian Institute of Jiangxi Province, China (Table 5). Genomic DNA was extracted from the leaf tissue of 46 cultivars by plant genomic DNA kit (TIANGEN Co. LTD) in accordance with the manufacturer's instructions and ran on $1 \%$ agarose gel for quality evaluation.

Determination of starch content in lotus root. Amylose, amylopectin and total starch content were measured both following a protocol of Williams V R et al ${ }^{34}$ based on at least three main roots selected from 46 lotus roots. Then the proportion of each component were calculated according to amylose content and amylopectin content. (Table 4).

Primer design and synthesis. The whole genome sequence of Chinese Lotus had already been made public ${ }^{35}$. Genomic DNA sequence of NnHXK was also obtained Gene ID:104592043. The NnGBSS gene had been cloned, and genomic DNA and cDNA sequence of NnGBSS were deposited to the GeneBank (GenBank accession no. FJ602702) ${ }^{18,36}$. Full length cDNA sequence of NnAGPS1 (GeneBank accession no. KJ476823) and NnAGPL (GeneBank accession no.KJ476824) were isolated and deposited to the GeneBank. Genomic DNA sequence of NnAGPS1 (GeneBank accession no. KJ476825) and NnAGPL (GeneBank accession no. KJ476826) were also obtained and sequenced ${ }^{21}$.

According to Genomic DNA and cDNA sequences of genes, primers were designed by using the software Primer Premier 5.0 meeting the following constraints: GC content of 40-60\%, 18-22 nucleotides in length, no secondary structure, and no consecutive tracts of a single nucleotide. All primers were synthesized by AuGCT Corporation (Beijing, Co. LTD).

PCR amplification and PAGE electrophoresis. The total volume of $25 \mu \mathrm{L}$ mixture containing $1 \mu \mathrm{L}$ Taq DNA polymerase, $1.4 \mu \mathrm{L}$ genomic DNA, $1.5 \mu \mathrm{L}$ forward primer $(20 \mu \mathrm{M}), 1.5 \mu \mathrm{L}$ reverse primer $(20 \mu \mathrm{M}), 2.5 \mu \mathrm{L}$ of

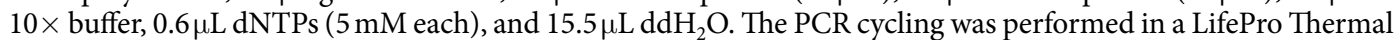
Cycler and amplifyed under the following conditions: $94^{\circ} \mathrm{C}$ for $5 \mathrm{~min}, 35$ cycles of $30 \mathrm{~s}$ at $94^{\circ} \mathrm{C}, 30 \mathrm{~s}$ at $51-63^{\circ} \mathrm{C}$, $45 \mathrm{~s}$ at $72^{\circ} \mathrm{C}$, and a final extension for $5 \mathrm{~min}$ at $72^{\circ} \mathrm{C}$.

The products of PCR amplified were mixed with loading buffer, denatured for $6 \mathrm{~min}$ at $95^{\circ} \mathrm{C}$, and utilized with a size standard marker of pBR322 DNA/Msp 1 (TIANGEN) to each lane. The products were analyzed by polyacrylamide gel (PAGE) electrophoresis on 6\% acrylamide, visualized by silver ion staining and photographed.

Association analysis. Based on the p/a(present/absent) of DNA bands (certain allele), all materials could be divided into two groups. The number of samples from each group were analysis by Excel double sample equal variance $\mathrm{t}$-test method. Based on the p/a amplified bands, we divided respectively amylose, amylopectin and total starch content into two groups. Two groups data were performed for correlation analysis.

Received: 24 October 2019; Accepted: 17 February 2020;

Published online: 06 March 2020

\section{References}

1. Guo, H. Cultivation of lotus (Nelumbo nucifera Gaertn. ssp. nucifera) and its utilization in China. Genetic Resources and Crop Evolution 56, 323-330 (2009).

2. Liangjun, L., Xiaodong, Z. \& Enchao, P. Relationship of starch synthesis with it's related enzymes' activities during rhizome development of lotus (Nelumbo nucifera Gaertn). Scientia Agricultura Sinica 11, 020 (2006).

3. Sridhar, K. \& Bhat, R. Lotus-A potential nutraceutical source. Journal of Agricultural Technology 3, 143-155 (2007).

4. Martin, C. \& Smith, A. M. Starch biosynthesis. The plant cell 7, 971 (1995).

5. Takeda, Y., Hizukuri, S., Takeda, C. \& Suzuki, A. Structures of branched molecules of amyloses of various origins, and molar fractions of branched and unbranched molecules. Carbohydrate Research 165, 139-145 (1987).

6. Wolosiuk, R. \& Pontis, H. Studies on sucrose synthetase: kinetic mechanism. Archives of biochemistry and biophysics 165, 140-145 (1974).

7. Varshney, R. K., Graner, A. \& Sorrells, M. E. Genomics-assisted breeding for crop improvement. Trends in plant science 10, 621-630 (2005).

8. Andersen, J. R. \& Lübberstedt, T. Functional markers in plants. Trends in plant science 8, 554-560 (2003).

9. Bagge, M., Xia, X. \& Lübberstedt, T. Functional markers in wheat. Current Opinion in Plant Biology 10, 211-216 (2007).

10. Liu, S. et al. Identification of two functional markers associated with drought resistance in maize. Molecular breeding 35, 53 (2015).

11. Rafalski, A. Applications of single nucleotide polymorphisms in crop genetics. Current opinion in plant biology 5, 94-100 (2002).

12. Yue, A. et al. Identification and development of a functional marker from 6-SFT-A2 associated with grain weight in wheat. Molecular Breeding 35, 63 (2015).

13. Murai, J., Taira, T. \& Ohta, D. Isolation and characterization of the three Waxy genes encoding the granule-bound starch synthase in hexaploid wheat. Gene 234, 71-79 (1999).

14. Chen, Y. et al. ISSR analysis of genetic diversity in sacred lotus cultivars. Aquatic Botany 89, 311-316 (2008).

15. Pan, L. et al. Development of novel EST-SSRs from sacred lotus (Nelumbo nucifera Gaertn) and their utilization for the genetic diversity analysis of $N$. nucifera. Journal of heredity 101, 71-82 (2009). 
16. Tian, H.-L., Xue, J.-H., Wen, J., Mitchell, G. \& Zhou, S.-L. Genetic diversity and relationships of lotus (Nelumbo) cultivars based on allozyme and ISSR markers. Scientia Horticulturae 116, 421-429 (2008).

17. Zhang, H.-Y., Dong, S.-T., Gao, R.-Q. \& Sun, Q.-Q. Starch accumulation and enzyme activities associated with starch synthesis in maize kernels. Agricultural Sciences in China 6, 808-815 (2007).

18. Lu, Y. et al. Cloning and characterization of the Wx gene encoding a granule-bound starch synthase in Lotus (Nelumbo nucifera Gaertn). Plant molecular biology reporter 30, 1210-1217 (2012).

19. Nakamura, T., Vrinten, P., Hayakawa, K. \& Ikeda, J. Characterization of a granule-bound starch synthase isoform found in the pericarp of wheat. Plant Physiology 118, 451-459 (1998).

20. Nakamura, T., Yamamori, M., Hirano, H., Hidaka, S. \& Nagamine, T. Production of waxy (amylose-free) wheats. Molecular and General Genetics MGG 248, 253-259 (1995).

21. Cheng, N. et al. Cloning and characterization of the genes encoding the small and large subunit of the ADP-glucose pyrophosphorylase in lotus (Nelumbo nucifera Gaertn). Acta physiologiae plantarum 37, 1734 (2015).

22. Preiss, J. Biosynthesis of bacterial and mammalian glycogen and plant starch synthesis and their regulation. Bioorganic chemistry: carbohydrates. Oxford University Press, Oxford, 59-114 (1999).

23. Smith-White, B. J. \& Preiss, J. Comparison of proteins of ADP-glucose pyrophosphorylase from diverse sources. Journal of Molecular Evolution 34, 449-464 (1992).

24. Newton, C. et al. Analysis of any point mutation in DNA. The amplification refractory mutation system (ARMS). Nucleic acids research 17, 2503-2516 (1989).

25. Okayama, H., Curiel, D. T., Brantly, M. L., Holmes, M. D. \& Crystal, R. G. Rapid, nonradioactive detection of mutations in the human genome by allele-specific amplification. The Journal of laboratory and clinical medicine 114, 105-113 (1989).

26. Sommer, S. S. et al. A novel method for detecting point mutations or polymorphisms and its application to population screening for carriers of phenylketonuria. Mayo Clinic Proceedings. Elsevier 64, 1361-1372 (1989).

27. Wu, D. Y., Ugozzoli, L., Pal, B. K. \& Wallace, R. B. Allele-specific enzymatic amplification of beta-globin genomic DNA for diagnosis of sickle cell anemia. Proceedings of the National Academy of Sciences 86, 2757-2760 (1989).

28. Iyer-Pascuzzi, A. S. \& McCouch, S. R. Functional markers for xa5-mediated resistance in rice (Oryza sativa, L.). Molecular Breeding 19, 291-296 (2007).

29. Fedorova, L. \& Fedorov, A. Introns in gene evolution. Genetica 118, 123-131 (2003).

30. He, X. et al. Characterization of phytoene synthase 1 gene (Psy1) located on common wheat chromosome $7 \mathrm{~A}$ and development of a functional marker. Theoretical and Applied Genetics 116, 213-221 (2008).

31. Beck, E. \& Ziegler, P. Biosynthesis and degradation of starch in higher plants. Annual review of plant biology 40, 95-117 (1989).

32. Chen, X., Salamini, F. \& Gebhardt, C. A potato molecular-function map for carbohydrate metabolism and transport. Theoretical and Applied Genetics 102, 284-295 (2001).

33. Winter, H. \& Huber, S. C. Regulation of sucrose metabolism in higher plants: localization and regulation of activity of key enzymes. Critical Reviews in plant sciences 19,31-67 (2000).

34. Williams, V. R., Wu, W.-T., Tsai, H. Y. \& Bates, H. G. Rice starch, varietal differences in amylose content of rice starch. Journal of Agricultural and Food Chemistry 6, 47-48 (1958).

35. Ming, R. et al. Genome of the long-living sacred lotus (Nelumbo nucifera Gaertn.). Genome biology 14, R41 (2013).

36. Gu, C. et al. Characterization of genes encoding granule-bound starch synthase in sacred lotus reveals phylogenetic affinity of Nelumbo to Proteales. Plant molecular biology reporter 31, 1157-1165 (2013).

\section{Acknowledgements}

This research was supported by National Key Technologies R\&D Program (No. 2012BAD27B01) and Technology Innovation Project of Hubei Provice of China (Major Program) (No. 2019ABA108).

\section{Author contributions}

T. Cheng performed some experiments, drew the figures and wrote the manuscript. X. Zheng and K. Xie provided materials. J. Liu and X. Zheng provided some scientific advices, correction. S. Jin and Y. Diao analyzed the results and provided some advices. J. Wang performed some experiment and outline the contents of the manuscript. Z. $\mathrm{Hu}$ and J. Wang approved the final manuscript. All authors have read and approved the manuscript in its final form.

\section{Competing interests}

The authors declare no competing interests.

\section{Additional information}

Supplementary information is available for this paper at https://doi.org/10.1038/s41598-020-60736-6.

Correspondence and requests for materials should be addressed to Z.H. or J.W.

Reprints and permissions information is available at www.nature.com/reprints.

Publisher's note Springer Nature remains neutral with regard to jurisdictional claims in published maps and institutional affiliations.

Open Access This article is licensed under a Creative Commons Attribution 4.0 International License, which permits use, sharing, adaptation, distribution and reproduction in any medium or format, as long as you give appropriate credit to the original author(s) and the source, provide a link to the Creative Commons license, and indicate if changes were made. The images or other third party material in this article are included in the article's Creative Commons license, unless indicated otherwise in a credit line to the material. If material is not included in the article's Creative Commons license and your intended use is not permitted by statutory regulation or exceeds the permitted use, you will need to obtain permission directly from the copyright holder. To view a copy of this license, visit http://creativecommons.org/licenses/by/4.0/.

(C) The Author(s) 2020 